\title{
Manual Compression Versus MANTA Device for Access Management After Impella Removal on the ICU
}

Florim Cuculi

Luzerner Kantonsspital

Philipp Burkart

Luzerner Kantonsspital

Giacomo Cioffi

Luzerner Kantonsspital

Federico Moccetti

Luzerner Kantonsspital

Mehdi Madanchi

Luzerner Kantonsspital

Thomas Seiler

Luzerner Kantonsspital

Stefanie Hess

Luzerner Kantonsspital

Mathias Wolfrum

Luzerner Kantonsspital

Magiliny Jeyarasa

Luzerner Kantonsspital

Sonja Meier

Luzerner Kantonsspital

Silvia Kuzmiakova

Luzerner Kantonsspital

Maani Hakimi

Luzerner Kantonsspital

Robert Seelos

Luzerner Kantonsspital

Richard Kobza

Luzerner Kantonsspital

Stefan Toggweiler

Luzerner Kantonsspital

Adrian Attinger 
Luzerner Kantonsspital

Matthias Bossard ( $\sim$ bossard.matthias@medportal.ca )

Luzerner Kantonsspital

\section{Research Article}

Keywords: Access closure, mechanical support, cardiogenic shock, Impella, Bleeding

Posted Date: January 3rd, 2022

DOI: https://doi.org/10.21203/rs.3.rs-1176070/v1

License: (c) (i) This work is licensed under a Creative Commons Attribution 4.0 International License. Read Full License 


\section{Abstract}

Objective: To compare the safety and efficacy of manual compression versus use of the MANTA ${ }^{\circledR}$ closure device for access management after Impella ${ }^{\circledR}$ removal on the intensive care unit (ICU).

Background: The number of patients treated with percutaneous left ventricular assist devices (pLVAD), namely Impella ${ }^{\circledR}$ and ECMO, for complex cardiac procedures or shock, is growing. However, removal of pLVAD and large bore arteriotomy closure among such patients on the ICU remains challenging, since it is associated with a high risk for bleeding and vascular complications.

Methods: Patients included in a prospective registry between 2017 and 2020 were analyzed. Bleeding and vascular access site complications were assessed and adjudicated according to VARC-2 criteria.

Results: We analyzed a cohort of 87 consecutive patients, who underwent access closure after Impella ${ }^{\circledR}$ removal on ICU by using either the MANTA ${ }^{\circledR}$ device or manual compression. The cohort's mean age was $66.1 \pm 10.7$ years and 76 patients ( $87 \%$ ) were recovering from CS. Mean support time was 40 hours (Interquartile range 24-69 hours). MANTA ${ }^{\circledR}$ was used in 31 patients (35.6\%) and manual compression was applied in 56 patients (64.4\%). Overall access related bleedings were significantly lower in the MANTA $^{\circledR}$ group (6.5\% versus $39.3 \%$ (odds ratio (OR) $0.10,95 \% \mathrm{Cl} 0.01-0.50 ; \mathrm{p}=0.001$ ), and there was no significant difference in vascular complications between the two groups $(p=0.55)$.

Conclusions: Our data suggests that the application of the MANTA ${ }^{\circledR}$ device directly on the ICU is safe. In addition, it seems to reduce access related bleeding without increasing the risk of vascular complications.

\section{Introduction}

During the last decade, the number of procedures and devices using large bore femoral vascular access has steadily grown. ${ }^{1}$ Such procedures include for instance transcatheter aortic valve replacement (TAVR), endovascular aneurysm repair (EVAR), but also percutaneous left ventricular assist devices (pLVAD). In this context, the catheter-based, continuous axial flow pumps Impella ${ }^{\circledR} 2.5$ and CP have become the preferred pLVADs for patients with profound cardiogenic shock (CS) or requiring high-risk percutaneous coronary interventions $(\mathrm{PCl}){ }^{2}$

Although, there is mounting data about strategies and devices for vascular closure in patients undergoing TAVR and EVAR, studies focusing on optimal non-surgical access closure in patients managed with the pLAVD Impella ${ }^{\circledR}$ remain scarce. In this context, one needs to take into account that Impella ${ }^{\circledR}$ removal on the intensive care unit (ICU) is particularly challenging and is prone to bleeding and vascular complications. ${ }^{3-5}$ Manual compression is often standard of care, but this is time-consuming and frequently semi-effective. ${ }^{5}$ 
For TAVR and EVAR, the MANTA® device, which represents a collagen-based, vascular closure device specifically designed for large bore femoral access site closure (12 to 24 French), truly facilitates access management, as highlighted by a series of recent studies. ${ }^{6-8}$ However, the utility and safety of this vascular closure device has not been systematically evaluated in patients requiring pLVAD support with an Impella ${ }^{\circledR}$. Especially patients, which require prolonged mechanical support and are transferred to the ICU after Impella ${ }^{\circledR}$ insertion in the catheter laboratory, might benefit from a device facilitating access closure while recovering.

Therefore, the aim of this analysis is to report the safety and efficacy of the MANTA® device compared to manual compression among patients, which undergo Impella ${ }^{\circledR}$ explantation while residing on the ICU.

\section{Methods}

\section{Study population}

From a prospective registry, we analyzed consecutive patients in cardiogenic shock (CS) or undergoing high-risk $\mathrm{PCl}$, who required prolonged hemodynamic circulatory support by Impella $\AA 2.5, \mathrm{CP}$ or $\mathrm{CP}$ Smart Assist (Abiomed Inc., Aachen, Germany) at the Heart Center of the Luzerner Kantonsspital between January 2017 and December 2020. The study conduct complies with the Declaration of Helsinki. Prospective data acquisition after enrollment was approved by the local and national ethics committee (EKNZ/ Swissethics, BASEC-ID 2019-00274, ClinicalTrials.gov Identifier. NCT04117230). Patients or their relatives provided written informed consent.

\section{Impella ${ }^{\circledR}$ device}

The catheter-based, continuous microaxial flow pumps Impella $\AA_{2}^{2.5, ~ C P ~ a n d ~ C P ~ S m a r t ~ A s s i s t ~ c a n ~ b e ~}$ implanted fully percutaneously, generally through the femoral artery. ${ }^{2,5}$ The Impella ${ }^{\circledR} 2.5$ and CP have an introducer sheath diameter of 12 and 14 French, respectively. ${ }^{2,5}$ As part of our clinical routine, we obtained a contrast angiogram in an ipsilateral projection to assess puncture height and anatomical suitability of the iliac and femoral arteries prior to Impella ${ }^{\circledR}$ insertion. Ultrasound guidance was used, whenever possible. However, considering the emergency setting of device implantation in CS patients ultrasound guidance was left to discretion of the treating physician. All patients were anticoagulated with unfractionated heparin (UFH) to achieve an activated clotting time (ACT) $>250$ s during PCl. Finally, the Impella ${ }^{\circledR}$ devices were inserted over a stiff 0.018 -inch guide wire ${ }^{2,5,9}$, advanced under fluoroscopy and positioned in a retrograde fashion across the aortic valve.

\section{Access closure strategies on ICU}

For access management after transfemoral implantation of an Impella ${ }^{\circledR}$ device, the following strategies for vascular closure on the ICU were utilized at our institution: (I) manual compression, (II) access closure using MANTA® 14F device, or (III) surgical removal in selected cases at high risk for bleeding or ischemic complications. Of note, pre-closure using the Perclose ProGlide ${ }^{\circledR}$ device (Abbott Vascular Inc., Santa 
Clara CA, U.S.A.) might only be applied in elective cases requiring short-term hemodynamic support at our institution, due to concerns about access site infections while leaving the sutures exposed on the ICU.

Our standard operating procedure for manual compression after transfemoral Impella ${ }^{\circledR}$ device removal recommends withholding heparin for at least two hours and until the ACT drops $<150 \mathrm{sec}$. Then, we applied manual compression for 10-20 minutes, followed by mechanical compression using the inflatable FemoStop ${ }^{\circledR}$ device (Abbott Vascular Inc., Santa Clara CA, U.S.A.) if needed, which allows gradual pressure release for 120-240 minutes.

The MANTA® device (Teleflex Inc., Morrisville, North Carolina) is a dedicated large-bore arteriotomy closure device, which may be used for access diameters ranging from 12-Fr to $25-\mathrm{F}$ in outer diameter. Two sizes are available (14- and 18-Fr). ${ }^{6-8}$ It comprises of a luminal resorbable polymer toggle and an extraluminal, resorbable, bovine collagen hemostatic plug. The toggle and plug are pulled together with a non-resorbable polyester suture topped by a radiopaque, stainless steel suture lock. We started using the MANTA $\AA$ device in March 2018 and all operators have extensive experience, with its deployment (including at least 10 supervised cases). ${ }^{7}$

In cases of (I) low puncture height (e.g. below the femoral bifurcation), (II) small vascular diameter ( $<6$ $\mathrm{mm}$ by visual estimation), and/or (III) prohibitive peripheral arterial disease (PAD), we did not deploy the MANTA ${ }^{\circledR}$ device. ${ }^{7}$ Before access closure with the MANTA® device, we aimed for an ACT < 180 sec. After extensive local disinfection sterile drapes were used to create a similar environment as in the cardiac catheterization laboratory. Over the Impella ${ }^{\circledR}$ introducer sheath side port, we inserted a 0.035 -inch guidewire, measured arterial access depth using the dedicated puncture depth locator and deployed the device. More details about the MANTA® device deployment can be found elsewhere. ${ }^{6}$ Manual compression or pressure bandages were only applied in cases if immediate hemostasis was not achieved.

\section{Data collection, outcomes and definitions}

Baseline demographics, procedural characteristics and outcome data were collected by study personal and entered in a dedicated database. All baseline angiograms of the iliac and femoral arteries were analyzed by a trained physician (P.B.) and quality of measurements was reviewed by a senior physician (A.T.). The minimal luminal diameter, degree of stenosis, severity of calcification, or other vascular abnormalities were determined. Measurement of puncture height was related to the center of the femoral head.

For the purpose of this study, we defined 'immediate hemostasis' as no relevant bleeding or additional medical measures (e.g. additional manual compression) directly after access closure or release of manual/mechanical compression. Strategy success was achieved when there were no signs of bleeding within 30 minutes after strategy termination. In case of MANTA® usage strategy success further included correct device deployment and no function failure of the device. Procedural and access site-related complications were assessed according to the Valve Academic Research Consortium (VARC) 2 criteria 
and adjudicated by two independent investigators (T.S. and M.M) ${ }^{10}$ In case of disagreement, consensus was reached through discussion with a senior physician (A.T.). Additionally, the following clinical outcomes within 30 days after the index event were analyzed: (I) minor or major bleeding according to the Bleeding Academic Research Consortium (BARC) criteria, (II) new myocardial infarction (MI), (III) stroke/ transient ischemic attack (TIA), (IV) repeat cardiogenic shock, (V) cardiovascular death and all-cause death. ${ }^{11}$

\section{Statistical analysis}

Continuous data are presented as mean \pm standard deviation (SD) or median (interquartile range (IQR)) as appropriate. Categorical variables are displayed as numbers (percentage). For comparison of variables the odds ratio (OR) and the $95 \%$ confidence interval $(\mathrm{Cl})$ were calculated. Furthermore, continuous variables were compared using the student's t-test or the Wilcoxon rank sum test. For statistical comparison between groups the Man-Whitney- $U$ test, chi-square test, Fishers exact test or students t-test were used. Categorical variables were compared using the chi-square test or the Fisher's exact test as appropriate. For the 30-day follow-up cox regression models were calculated. A p-value $<0.05$ was considered statistically significant. The statistical analyses were conducted with Stata/SE 16.1 (StataCorp, College Station, Texas, USA).

\section{Results}

\section{Patient and procedural characteristics}

Overall, 170 patients required mechanical circulatory support with an Impella ${ }^{\circledR}$ device between January 2017 and December 2020. Of those, in consecutive 87 patients (51\%) the Impella ${ }^{\circledR}$ device was removed directly on the ICU (Figure 1). The mean age was $66.1 \pm 10.6$ years, $21(24 \%)$ were females, the majority was recovering from CS (87\%). Totally, 51 patients $(58.6 \%)$ had initially presented with a STEMI. The baseline characteristics are depicted in Table 1. The MANTA® device was used in 31 patients $(35.6 \%)$ and manual compression was applied in 56 patients (64.4\%). Of note, both groups were balanced in terms of comorbidities, including the presence of PAD (overall 13.8\%), as highlighted in Table 1. Importantly, minimal femoral arterial diameter was similar in both groups $(p=0.26)$. The larger Impella ${ }^{\circledR}$ CP was implanted in most patients (95.4\%) and median hemodynamic support time was 39.8 hours (IQR 23.6-68.9 hours). However, hemodynamic support time was somewhat longer in the manual compression group than in the MANTA® group (45.0 (IQR 28.5-75.1 hours) versus 34.5 hours (IQR 12.548.1 hours); $p=0.036)$. The anatomical and procedural characteristics are presented in Table 2. 
Table 1

Baseline demographics grouped according to the access closure strategy on ICU

\begin{tabular}{|c|c|c|c|c|}
\hline & $\begin{array}{l}\text { Overall } \\
(n=87)\end{array}$ & $\begin{array}{l}\text { MANTA® } \\
\text { device } \\
(n=31)\end{array}$ & $\begin{array}{l}\text { Manual } \\
\text { compression } \\
(n=56)\end{array}$ & $\begin{array}{l}P \text { - } \\
\text { value }\end{array}$ \\
\hline Age (years) & $66.1 \pm 10.7$ & $64.7 \pm 9.1$ & $66.9 \pm 11.5$ & 0.37 \\
\hline Female (\%) & $21(24.1)$ & $6(19.4)$ & $15(26.8)$ & 0.44 \\
\hline $\mathrm{BMI}\left(\mathrm{kg} / \mathrm{m}^{2}\right)$ & $26.1 \pm 4.0$ & $26.3 \pm 4.6$ & $26.0 \pm 3.7$ & 0.74 \\
\hline $\begin{array}{l}\text { Cardiac arrest prior to } \\
\text { admission }\end{array}$ & $45(51.7)$ & $12(38.7)$ & $33(58.9)$ & 0.07 \\
\hline Cause of CS. & & & & 0.53 \\
\hline STEMI & $51(58.6)$ & $16(51.6)$ & $35(62.5)$ & \\
\hline NSTEMI/ unstable AP & $21(24.1)$ & $8(25.8)$ & $13(23.2)$ & \\
\hline No ACS & $15(17.2)$ & $7(22.6)$ & $8(14.3)$ & \\
\hline \multicolumn{5}{|l|}{ Comorbidities: } \\
\hline Previous PCl & $17(19.5)$ & $9(29.0)$ & $8(14.3)$ & 0.09 \\
\hline Previous MI & $14(16.1)$ & $6(19.4)$ & $8(14.3)$ & 0.54 \\
\hline Previous CABG & $8(9.2)$ & $2(6.5)$ & $6(10.7)$ & 0.51 \\
\hline PAD & $12(13.8)$ & $6(19.4)$ & $6(10.7)$ & 0.26 \\
\hline Previous stroke / TIA & $6(6.9)$ & $4(12.9)$ & $2(3.6)$ & 0.10 \\
\hline History of heart failure & $3(3.4)$ & $0(0)$ & $3(5.4)$ & 0.19 \\
\hline Diabetes & $27(31.0)$ & $14(45.2)$ & $13(23.2)$ & 0.039 \\
\hline Arterial hypertension & $49(56.3)$ & $19(61.3)$ & $30(53.6)$ & 0.48 \\
\hline Dyslipidemia & $36(41.4)$ & $14(45.2)$ & $22(39.3)$ & 0.59 \\
\hline Obesity (BMI >30) & $15(17.2)$ & $8(25.8)$ & $7(12.5)$ & 0.20 \\
\hline Current smoking & $18(20.7)$ & $9(29)$ & $9(16.1)$ & 0.15 \\
\hline Chronic kidney disease $^{+}$ & $11(12.6)$ & $4(12.9)$ & $7(12.5)$ & 0.95 \\
\hline Preexisting anemia & $4(4.6)$ & $2(6.5)$ & $2(3.6)$ & 0.61 \\
\hline Initial LVEF & $25 \pm 8$ & $25 \pm 6$ & $25 \pm 9$ & 0.77 \\
\hline LVEDP (mmHg) & $32 \pm 9$ & $34 \pm 9$ & $32 \pm 8$ & 0.46 \\
\hline
\end{tabular}




\begin{tabular}{|c|c|c|c|c|}
\hline & $\begin{array}{l}\text { Overall } \\
(n=87)\end{array}$ & $\begin{array}{l}\text { MANTA® } \\
\text { device } \\
(n=31)\end{array}$ & $\begin{array}{l}\text { Manual } \\
\text { compression } \\
(n=56)\end{array}$ & $\begin{array}{l}P \text { - } \\
\text { value* }\end{array}$ \\
\hline \multicolumn{5}{|l|}{ Laboratory parameters ${ }^{\ddagger}:$} \\
\hline Peak lactate (mmol/L) & $2.6(1.7-4.5)$ & $2.4(1.6-3.2)$ & $2.7(1.9-5)$ & 0.11 \\
\hline $\begin{array}{l}\text { eGFR (CKD-EPI) } \\
\left(\mathrm{ml} / \mathrm{min} / 1.73 \mathrm{~m}^{2}\right)\end{array}$ & $72(52-91)$ & $74.5(63.5-91)$ & $67(48-90)$ & 0.16 \\
\hline Peak troponin T (ng/L) & $\begin{array}{l}4323(1547- \\
12384)\end{array}$ & $\begin{array}{l}2901 \text { (1547- } \\
9630)\end{array}$ & $\begin{array}{l}4907(1519- \\
15481)\end{array}$ & 0.29 \\
\hline Peak CK (IU/L) & $3002(811-6078)$ & $\begin{array}{l}1754(779- \\
5027)\end{array}$ & $3445(992-6531)$ & 0.29 \\
\hline $\begin{array}{l}\text { Hemoglobin prior to closure } \\
(\mathrm{g} / \mathrm{L})\end{array}$ & $97.8 \pm 18.8$ & $105 \pm 20.1$ & $93.8 \pm 16.9$ & 0.007 \\
\hline Thrombocytes (G/L) & $160 \pm 89$ & $188 \pm 104$ & $145 \pm 77$ & 0.036 \\
\hline Anti-Xa level (U/mL) & $0.47(0.23-0.83)$ & $\begin{array}{l}0.54(0.21- \\
0.89)\end{array}$ & $0.47(0.23-0.80)$ & 0.73 \\
\hline \multicolumn{5}{|l|}{$\begin{array}{l}\text { Type of Intervention } \\
\text { performed: }\end{array}$} \\
\hline PCl performed & 75 (86.2) & $26(83.9)$ & $49(87.5)$ & 0.64 \\
\hline CABG performed & $3(3.4)$ & $2(6.5)$ & $1(1.8)$ & 0.25 \\
\hline \multicolumn{5}{|l|}{ Antithrombotics§ } \\
\hline Aspirin & 78 (89.7) & $26(83.9)$ & $52(92.9)$ & 0.19 \\
\hline Ticagrelor & $56(64.4)$ & $17(54.8)$ & $39(69.6)$ & 0.16 \\
\hline Cangrelor & $9(10.3)$ & $7(22.6)$ & $2(3.6)$ & 0.006 \\
\hline Prasugrel & $1(1.1)$ & $0(0)$ & $1(1.8)$ & 0.45 \\
\hline Clopidogrel & $9(10.3)$ & $3(9.7)$ & $6(10.7)$ & 0.87 \\
\hline GPIIbllla inhibitors & $9(10.3)$ & $1(3.2)$ & $8(14.3)$ & 0.10 \\
\hline Unfractionated heparin & $67(77.0)$ & $28(90.3)$ & $39(69.6)$ & 0.029 \\
\hline DOACs & $2(2.3)$ & $0(0)$ & $2(3.6)$ & 0.28 \\
\hline
\end{tabular}




\begin{tabular}{|c|c|c|c|}
\hline $\begin{array}{l}\text { Overall } \\
(n=87)\end{array}$ & $\begin{array}{l}\text { MANTA® } \\
\text { device } \\
(n=31)\end{array}$ & $\begin{array}{l}\text { Manual } \\
\text { compression } \\
(n=56)\end{array}$ & $\begin{array}{l}P \text { - } \\
\text { value* }\end{array}$ \\
\hline \multicolumn{4}{|c|}{$\begin{array}{l}\text { Data are median (interquartile range) or number (percentage), as appropriate. AP = Angina pectoris; } \\
\text { BMI = Body Mass Index; CS = Cardiogenic shock; STEMI =ST-segment-elevation acute myocardial } \\
\text { infarction; NSTEMI = Non-ST-segment-elevation myocardial infarction; PCI = Percutaneous coronary } \\
\text { intervention; MI = Myocardial infarction; PAD = Peripheral arterial disease; CABG = Coronary artery } \\
\text { bypass grafting; TIA = Transient Ischemic Attack; Hb = Hemoglobin; LVEF = Left ventricular ejection } \\
\text { fraction; LVEDP = Left ventricular end diastolic pressure; eGFR (CKD-EPI) = Estimated glomerular } \\
\text { filtration rate (Chronic Kidney Disease Epidemiology Collaboration); CK = Creatine Kinase; DOAC = } \\
\text { Direct Oral Anticoagulant Agents }\end{array}$} \\
\hline \multicolumn{4}{|c|}{ *P values were based on Fisher's exact, Man-Whitney-U test or student's t-test, as appropriate. } \\
\hline \multicolumn{4}{|c|}{${ }^{\dagger}$ Chronic kidney disease was defined as a preexisting eGFR $<45 \mathrm{ml} / \mathrm{min} / \mathrm{m}^{2}$} \\
\hline \multicolumn{4}{|c|}{$\begin{array}{l}\ddagger \text { Values of laboratory parameters prior to access closure are represented (either first or peak, as } \\
\text { appropriate). }\end{array}$} \\
\hline $\begin{array}{l}\S \text { This comprised antithrombotics, which } \\
\text { prior to device removal. }\end{array}$ & dministered & npella ${ }^{\circledR}$ implan & and/ or \\
\hline
\end{tabular}


Table 2

Access and device-related characteristics

\begin{tabular}{|c|c|c|c|c|}
\hline & $\begin{array}{l}\text { Overall } \\
(n=87)\end{array}$ & $\begin{array}{l}\text { MANTA® } \\
\text { device } \\
(n=31)\end{array}$ & $\begin{array}{l}\text { Manual } \\
\text { compression } \\
(n=56)\end{array}$ & $\begin{array}{l}P \text { - } \\
\text { value* }\end{array}$ \\
\hline \multicolumn{5}{|l|}{ Vessel characteristics: } \\
\hline $\begin{array}{l}\text { Minimal iliac artery diameter } \\
(\mathrm{mm})\end{array}$ & $7.8 \pm 1.7$ & $8.16 \pm 1.8$ & $7.5 \pm 1.6$ & 0.17 \\
\hline $\begin{array}{l}\text { Minimal femoral artery diameter } \\
(\mathrm{mm})\end{array}$ & $7.2 \pm 1.7$ & $7.5 \pm 2.0$ & $7.0 \pm 1.5$ & 0.26 \\
\hline None to mild calcification & $44(50.6)$ & $17(54.8)$ & $27(48.2)$ & 0.63 \\
\hline Moderate calcification & $12(13.8)$ & $7(22.6)$ & $5(8.9)$ & 0.18 \\
\hline Severe calcification & $8(9.2)$ & $2(6.5)$ & $6(10.7)$ & 0.33 \\
\hline Single site access & $13(14.9)$ & $11(35.5)$ & $2(3.6)$ & $<0.001$ \\
\hline \multicolumn{5}{|l|}{ Impella devices } \\
\hline 2.5 & $4(4.6)$ & $0(0)$ & $4(7.1)$ & 0.12 \\
\hline CP / Smart Assist & $83(95.4)$ & $31(100)$ & $52(92.9)$ & 0.12 \\
\hline \multicolumn{5}{|l|}{ Impella implantation $^{\dagger}$} \\
\hline Easy & $44(50.6)$ & $14(45.2)$ & $30(53.6)$ & 0.24 \\
\hline Some difficulties & $28(32.2)$ & $12(38.7)$ & $16(28.6)$ & 0.47 \\
\hline Very challenging & $8(9.2)$ & $4(12.9)$ & $4(7.1)$ & 0.44 \\
\hline Implantation under resuscitation & $10(11.5)$ & $2(6.5)$ & $8(14.3)$ & 0.26 \\
\hline pLVAD support time (hours) & $\begin{array}{l}39.8(23.6- \\
68.9)\end{array}$ & $\begin{array}{l}34.5(12.5- \\
48.1)\end{array}$ & $45.0(28.5-75.1)$ & 0.036 \\
\hline \multicolumn{5}{|c|}{$\begin{array}{l}\text { Data are median (interquartile range) or number (percentage), as appropriate. pLVAD = percutaneous } \\
\text { left ventricular assist device }\end{array}$} \\
\hline \multicolumn{5}{|c|}{${ }^{*} \mathrm{P}$ values were based on Man-Whitney-U test or t-test as appropriate. } \\
\hline
\end{tabular}

\section{Access related bleeding and vascular complications}


The Figure 2 summarizes the main results. Immediate hemostasis was significantly more often achieved with the MANTA® device ( $90.3 \%$ versus $60.7 \%$; odds ratio (OR) $5.76,95 \%$ confidence interval (Cl) 1.46 $32.67 ; p=0.005)$. However, overall strategy success rate was high $(94.3 \%)$ and there was no significant difference between groups $(p=0.55)$. Overall, any access related complications were significantly lower in the MANTA $\AA$ compared to the manual compression group $(19.4 \%$ versus $46.4 \%$, OR $0.28,95 \% \mathrm{Cl} 0.08$ $0.84 ; p=0.012$ ). Also, bleeding complications according to VARC-2 criteria were significantly lower in the MANTA $\circledast$ group (6.5\% versus $39.3 \%$, OR $0.10,95 \% 95 \% \mathrm{Cl} 0.01-0.5, \mathrm{p}=0.001)$. Regarding vascular complications, there was no difference between the two groups $(16.1 \%$ versus $21.4 \%$, OR $0.70, \mathrm{Cl} 0.17$ $2.47, p=0.55)$. Access related outcomes are depicted in Table 3 . The narratives of major complications are presented in Supplemental Table 1. 
Table 3

Access related outcomes after Impella ${ }^{\circledR}$ removal

\begin{tabular}{|c|c|c|c|c|c|}
\hline Access-related outcomes & $\begin{array}{l}\text { Overall } \\
(n=87)\end{array}$ & $\begin{array}{l}\text { MANTA® } \\
\text { device } \\
(n=31)\end{array}$ & $\begin{array}{l}\text { Manual } \\
\text { compression } \\
(n=56)\end{array}$ & OR $(95 \% \mathrm{Cl})^{*}$ & $\begin{array}{l}P- \\
\text { value }^{+}\end{array}$ \\
\hline Immediate hemostasis ${ }^{\ddagger}$ & $\begin{array}{l}62 \\
(71.3)\end{array}$ & $28(90.3)$ & $34(60.7)$ & $\begin{array}{l}5.76(1.46- \\
32.67)\end{array}$ & 0.0049 \\
\hline $\begin{array}{l}\text { Successful MANTA® } \\
\text { deployment } \S\end{array}$ & & $29(93.5)$ & - & - & - \\
\hline Strategy success" & $\begin{array}{l}82 \\
(94.3)\end{array}$ & $29(93.5)$ & $53(94.6)$ & $\begin{array}{l}0.54(0.04- \\
7.97)\end{array}$ & 0.55 \\
\hline $\begin{array}{l}\text { Any access-related adverse } \\
\text { outcome }\end{array}$ & $\begin{array}{l}32 \\
(36.8)\end{array}$ & $6(19.4)$ & $26(46.4)$ & $\begin{array}{l}0.276(0.08- \\
0.84)\end{array}$ & 0.012 \\
\hline \multicolumn{6}{|l|}{ VARC Bleeding: } \\
\hline Overall & $\begin{array}{l}24 \\
(27.6)\end{array}$ & $2(6.5)$ & $22(39.3)$ & $\begin{array}{l}0.106(0.01- \\
0.50)\end{array}$ & 0.001 \\
\hline Minor bleeding (BARC 2) & $\begin{array}{l}16 \\
(18.4)\end{array}$ & $1(3.2)$ & $15(26.8)$ & $\begin{array}{l}0.091(0.002- \\
0.67)\end{array}$ & 0.006 \\
\hline Major bleeding (BARC 3a) & $7(8.0)$ & $1(3.2)$ & $6(10.7)$ & $\begin{array}{l}0.277(0.005- \\
2.49)\end{array}$ & 0.22 \\
\hline $\begin{array}{l}\text { Life-threatening (BARC } \\
3 \mathrm{~b} / 5)\end{array}$ & $1(1.1)$ & $0(0)$ & $1(1.8)$ & - & 0.45 \\
\hline \multicolumn{6}{|l|}{$\begin{array}{l}\text { VARC vascular } \\
\text { complications: }\end{array}$} \\
\hline Overall & $\begin{array}{l}17 \\
(19.5)\end{array}$ & $5(16.1)$ & $12(21.4)$ & $\begin{array}{l}0.70(0.17- \\
2.47)\end{array}$ & 0.55 \\
\hline Minor complications & $3(3.4)$ & $2(6.5)$ & $1(1.8)$ & $\begin{array}{l}3.79(0.19- \\
227.73)\end{array}$ & 0.25 \\
\hline Major complications & $\begin{array}{l}14 \\
(16.1)\end{array}$ & $3(9.7)$ & $11(19.6)$ & $\begin{array}{l}0.44(0.07- \\
1.88)\end{array}$ & 0.22 \\
\hline Urgent vascular surgery & $7(8.0)$ & $3(9.7)$ & $4(7.1)$ & $\begin{array}{l}1.39(0.19- \\
8.84)\end{array}$ & 0.67 \\
\hline \multicolumn{6}{|c|}{$\begin{array}{l}\text { Data are median (interquartile range) or number (percentage), as appropriate. BARC = Bleeding } \\
\text { Academic Research Consortium; } \mathrm{Cl}=\text { Confidence intervals; } \mathrm{OR}=\text { Odds ratio; VARC = Valve Academic } \\
\text { Research Consortium }\end{array}$} \\
\hline \multicolumn{6}{|c|}{ * OR and $\mathrm{Cl}$ was calculated using manual compression as control group } \\
\hline
\end{tabular}




\begin{tabular}{|c|c|c|c|c|c|}
\hline Access-related outcomes & $\begin{array}{l}\text { Overall } \\
(n=87)\end{array}$ & $\begin{array}{l}\text { MANTA® } \\
\text { device } \\
(n=31)\end{array}$ & $\begin{array}{l}\text { Manual } \\
\text { compression } \\
(n=56)\end{array}$ & OR $(95 \% C l)^{*}$ & $\begin{array}{l}P- \\
\text { value }^{t}\end{array}$ \\
\hline \multicolumn{6}{|c|}{$\begin{array}{l}\text { ₹Immediate hemostasis was defined as a state of no relevant bleeding or oozing with no additional } \\
\text { medical action needed directly after access closure. Only } 86 \text { patients could be included for this, } 1 \\
\text { patient in the manual compression group could not be evaluated. }\end{array}$} \\
\hline \multicolumn{6}{|c|}{$\begin{array}{l}\text { § Successful MANTA® deployment was defined as a correct release and placement of the MANTA® } \\
\text { toggle and plug and was evaluated at each closure by the responsible operator. }\end{array}$} \\
\hline \multicolumn{6}{|c|}{$\begin{array}{l}\text { "Strategy success was achieved when there were no signs of bleeding within } 30 \text { minutes after } \\
\text { Impellaß removal and, in case of MANTA® usage, if there was correct deployment of the vascular } \\
\text { closure device and no complications had occurred. }\end{array}$} \\
\hline
\end{tabular}

\section{Clinical outcomes at 30-days}

No additional vascular complications occurred during the follow-up period. Most importantly we did not encounter any relevant access site related infections. $10.3 \%$ of patients suffered from septic infections. However, infectious complications were equally distributed between the two groups $(12.9 \%$ versus $8.9 \%$, hazard ratio $(\mathrm{HR}) 0.67,95 \% \mathrm{Cl} 0.18-2.52, \mathrm{p}=0.56$ ). Also, there was no significant difference between allcause mortality $(22.6 \%$ versus $19.6 \%, \mathrm{HR} 0.87, \mathrm{Cl} 0.34-2.26, \mathrm{p}=0.78)$. 30 -day outcomes are reported in Table 4. 
Table 4

Clinical outcomes at 30-day

\begin{tabular}{|c|c|c|c|c|c|}
\hline & $\begin{array}{l}\text { Overall } \\
(n=87)\end{array}$ & $\begin{array}{l}\text { MANTA® } \\
\text { device } \\
(n=31)\end{array}$ & $\begin{array}{l}\text { Manual } \\
\text { compression } \\
(n=56)\end{array}$ & & $\begin{array}{l}P- \\
\text { value }^{t}\end{array}$ \\
\hline $\begin{array}{l}\text { Any packed RBC } \\
\text { transfusion }\end{array}$ & $2(0-5)$ & $0(0-4)$ & $3(1-5)$ & & 0.004 \\
\hline ICU stay (days) & $\begin{array}{l}5(3- \\
10)\end{array}$ & $4(3-8)$ & $6(4-11)$ & & 0.14 \\
\hline $\begin{array}{l}\text { Duration of hospital stay } \\
\text { (days) }\end{array}$ & $\begin{array}{l}11(7- \\
20)\end{array}$ & $10.5(7-18)$ & $11(7-20.5)$ & & 0.91 \\
\hline $\begin{array}{l}\text { Clinical outcomes at } 30 \text { - } \\
\text { days }\end{array}$ & & & & $\mathrm{HR}(95 \% \mathrm{Cl})$ & \\
\hline Minor bleeding (BARC 2$)^{\star}$ & $\begin{array}{l}14 \\
(16.1)\end{array}$ & $6(19.4)$ & $8(14.3)$ & $\begin{array}{l}0.81(0.23- \\
2.86)\end{array}$ & 0.74 \\
\hline $\begin{array}{l}\text { Major bleeding (BARC 3/ } \\
5)^{\star}\end{array}$ & $\begin{array}{l}37 \\
(42.5)\end{array}$ & $13(41.9)$ & $24(42.9)$ & $\begin{array}{l}0.87(0.38- \\
2.02)\end{array}$ & 0.75 \\
\hline New Ml & $5(5.7)$ & $4(12.9)$ & $1(1.8)$ & $\begin{array}{l}0.13(0.01- \\
1.18)\end{array}$ & 0.07 \\
\hline Stroke / TIA & $6(6.9)$ & $2(6.5)$ & $4(7.1)$ & $\begin{array}{l}1.08(0.2- \\
5.91)\end{array}$ & 0.92 \\
\hline New heart failure & $\begin{array}{l}11 \\
(12.6)\end{array}$ & $7(22.6)$ & $4(7.1)$ & $\begin{array}{l}0.26(0.06- \\
1.03)\end{array}$ & 0.054 \\
\hline Repeat CS & $7(8.0)$ & $3(9.7)$ & $4(7.1)$ & $\begin{array}{l}0.71(0.16- \\
3.19)\end{array}$ & 0.65 \\
\hline Sepsis & $\begin{array}{l}9 \\
(10.3)\end{array}$ & $4(12.9)$ & $5(8.9)$ & $\begin{array}{l}0.68(0.18- \\
2.52)\end{array}$ & 0.56 \\
\hline Cardiovascular death & $\begin{array}{l}18 \\
(20.7)\end{array}$ & $7(22.6)$ & $11(19.6)$ & $\begin{array}{l}0.87(0.34- \\
2.26)\end{array}$ & 0.78 \\
\hline All-cause death & $\begin{array}{l}18 \\
(20.7)\end{array}$ & $7(22.6)$ & $11(19.6)$ & $\begin{array}{l}0.87(0.34- \\
2.26)\end{array}$ & 0.78 \\
\hline \multicolumn{6}{|c|}{$\begin{array}{l}\text { Data are median (interquartile range) or number (percentage), as appropriate. BARC = Bleeding } \\
\text { Academic Research Consortium; CS = Cardiogenic shock; HR = Hazard ratio; ICU = Intensive care unit; } \\
\text { MI = Myocardial Infarction; RBC = Red blood cells; TIA = Transient ischemic attack }\end{array}$} \\
\hline
\end{tabular}


Our study suggests that using the MANTA $®$ device for femoral arteriotomy closure after Impella ${ }^{\circledR}$ device removal in the ICU setting is safe and effective. Compared to manual compression, we observed a lower incidence of access-related bleeding complications and similar vascular complication rates. Albeit earlier studies by our and other groups have indicated the efficacy and safety of the MANTA® closure device after pLVAD removal, none of them investigated its use outside of a dedicated area such as the cardiac catheterization laboratory or the operating room. ${ }^{7,12}$ To the best of our knowledge this is the first report describing the use of the MANTA® device for large bore access-site management on the ICU.

As widely known, 30-day mortality in CS patients remains high (up to 50\%) despite immediate treatment and hemodynamic support in the form of a pLVAD. ${ }^{13}$ Patients recovering from CS and requiring mechanical support usually represent an extreme risk patient cohort, which is prone to complications. It is well known that bleeding is associated with a twofold increase in mortality rates in patients with acute myocardial infarction complicated by cardiogenic shock. ${ }^{14}$ Additionally, intrahospital transportation has been shown to increase the complication rate of critically ill patients. ${ }^{15}$ Consequently, adverse events related to poor femoral access management puts CS patients at great risk and the fashion as well as the locality of device removal need to be diligently planned and executed.

In line with previous reports, our study demonstrates that the MANTA® closure device is safe and efficient for large-bore vessel closure. 7,16 Vascular complications after pLVAD removal remain a challenge, even for experienced operators. Despite of using the MANTA® closure device on the ICU, vascular complication rates observed in our cohort (19.5\%) were comparable to other studies describing outcomes after pLVAD removal in dedicated areas (4-17\%). ${ }^{7,17-20}$ But one also needs to take in account that the majority of our patients underwent insertion of an Impella CP device in an emergent setting or even under ongoing resuscitation due to cardiogenic shock. Such a setting not just complicates the arteriotomy and device implantation, but it may be directly linked to a higher bleeding and vascular complication risk. Taken together, the rate of acute limb ischemia (2.3\%) was relatively low in our cohort compared to prior studies analyzing outcomes after pLVAD placement (up to $13 \%) .{ }^{18,19}$

When comparing the two strategies for access management, we observed no increased incidence of vascular complications after application of the MANTA® device when compared to manual compression. On the contrary, the rate of access-related adverse outcome was twice as high in the manual compression group (Table 3). One also needs to take in account, that we did not identify any MANTA® related access site or systemic infections in our cohort. Consequently, there was also no difference in the occurrence of sepsis or severe infections at 30-day follow-up between the two groups (Table 4) This is important since patients in the critical care settings are more susceptible to nosocomial infections. ${ }^{21}$ Thus, our data may implicate that the risk for infectious complications with the delivery of the MANTA® closure device in the ICU setting are very low, if carefully executed.

Although, other vascular closure device systems are available for large bore access site management, the MANTA $®$ closure device entails major advantages in the setting of CS patients. In contrast to percutaneous suture-mediated closure devices such as the Perclose ProGlide® SMC System or the 
Prostar XL® percutaneous vascular surgical system (Abbott Cardiovascular, Plymouth, MN), which may only be used in a pre-close fashion, post-procedural closure by the MANTA® closure device allows immediate implantation of the PLVAD without delaying the potentially lifesaving PCI in these acutely ill patients.

Finally, device success rate in the MANTA® group was high (94\%). However, to achieve device success, pre-assessment by angiography or sonography of the femoral artery before pLVAD implantation appears key. ${ }^{7}$ If feasible, we assess the arterial diameter, degree of calcification and the site of arterial puncture prior to Impella ${ }^{\circledR}$ implantation. Visualization of the femoral artery can quickly be done before pLVAD implantation and certainly helps identifying patient with an unsuitable anatomy and therefore decreases the risk for vascular complications without delaying the procedure itself.

Manual compression for large-bore arterial access management after pLVAD removal is time-consuming, causes discomfort for patients and access-site bleeding control might not be achieved. ${ }^{22}$ In contrast, the reduction in bleeding complications and faster mobilization after use of MANTA® could potentially translate into shortened ICU stays and moreover mitigate the patients' morbidity risk. However, this needs to be further investigated.

\section{Limitations}

The results of the present study need to be interpreted in the light of the following limitations: Firstly, this was an observational, non-randomized single center study. Considering the small number of patients, the study was underpowered to identify predictors of worse outcome after MANTA® device closure. Secondly, although the Impella ${ }^{\circledR}$ removal and access site closure was performed by experienced interventional cardiologists, a learning curve might have had some impact on outcomes of patients in the MANTA ${ }^{\circledR}$ group. Thirdly, standard operating procedures at our institution regarding the use of GP-Ilb/IIlainhibitors have changed during the period of observation. Based on our experience and on published evidence showing the risk of GP-Ilb/Illa-inhibitors in the setting of large bore access, we completely stopped using these medications in patients with pLVADs.

\section{Conclusions}

With respect to the growing number of patients treated with percutaneous left ventricular assist devices (pLVAD), for complex cardiac procedures, heart failure and shock, there is a need for enhanced strategies for access management. In this context, we studied the safety and efficacy of the novel MANTA® closure device compared to manual compression for femoral arteriotomy closure after Impella ${ }^{\circledR}$ removal in the ICU setting. Our data suggest that the application of the MANTA® device can be safely used for this purpose in selected patients. Moreover, it seems to reduce access related bleedings without increasing the risk of vascular complications.

\section{Declarations}




\section{Funding}

This is an independent investigator-initiated study.

\section{Disclosures}

MB has received speaker fees from Abiomed. ST is a consultant for Teleflex, Shockwave, atHeart, Medira, VeoSource, a consultant and proctor for Boston Scientific, Medtronic and Biosensors, a proctor for Abbott vascular, has received institutional grant support from Boston Scientific and Fumedica and holds equity in Hi-D Imaging. RK has received institutional grant support from Abbott, Biotronik, Biosense Webster, Boston Scientific, Medtronic and SIS Medical. FC has received speaker fees and research grants from Abiomed, Abbott Vascular and SIS Medical. None of the other authors had any relevant conflicts of interest.

\section{Authors contributions}

$\mathrm{MB}, \mathrm{PB}, \mathrm{ST}, \mathrm{AA}$, and $\mathrm{FC}$ conceived and designed the analysis. PB, GC, MM, TS, SH, MJ and SM collected the data. FM, MW, SK, ST, MH and RS contributed data. MB, PB, MM and AA performed the analysis. MB, PB, AA and FC wrote the paper. GC, MM, TS, SH, MJ, SM, FM, MW, SK, RK, ST, MH and RS edited and reviewed the paper. MB, FC and RK supervised the project and organized research staff to support the project / analysis. SM supervised the data collection. AA, MM and TS reviewed the outcomes / study endpoints. GC drafted the illustration (graphical abstract). All authors had reviewed and approved the final version of the manuscript.

\section{References}

1. Kaki, A. et al. Access and closure management of large bore femoral arterial access. J Interv Cardiol 31, 969-977, doi:10.1111/joic.12571 (2018).

2. Balthazar, T. et al. Managing Patients With Short-Term Mechanical Circulatory Support: JACC Review Topic of the Week. J Am Coll Cardiol 77, 1243-1256, doi:10.1016/j.jacc.2020.12.054 (2021).

3. Schrage, B. et al. Impella Support for Acute Myocardial Infarction Complicated by Cardiogenic Shock. Circulation 139, 1249-1258, doi:10.1161/CIRCULATIONAHA.118.036614 (2019).

4. Ouweneel, D. M. et al. Percutaneous Mechanical Circulatory Support Versus Intra-Aortic Balloon Pump in Cardiogenic Shock After Acute Myocardial Infarction. J Am Coll Cardio/ 69, 278-287, doi:10.1016/j.jacc.2016.10.022 (2017).

5. Burzotta, F. et al. Impella ventricular support in clinical practice: Collaborative viewpoint from a European expert user group. Int J Cardiol 201, 684-691, doi:10.1016/j.ijcard.2015.07.065 (2015).

6. Wood, D. A. et al. Pivotal Clinical Study to Evaluate the Safety and Effectiveness of the MANTA Percutaneous Vascular Closure Device. Circ Cardiovasc Interv 12, e007258, doi:10.1161/CIRCINTERVENTIONS.119.007258 (2019). 
7. Moccetti, F. et al. Insights From a Multidisciplinary Introduction of the MANTA Vascular Closure Device. JACC Cardiovasc Interv 12, 1730-1736, doi:10.1016/j.jcin.2019.06.049 (2019).

8. Van Mieghem, N. M. et al. Percutaneous Plug-Based Arteriotomy Closure Device for Large-Bore Access: A Multicenter Prospective Study. JACC Cardiovasc Interv 10, 613-619, doi:10.1016/j.jcin.2016.12.277 (2017).

9. Succar, L., Sulaica, E. M., Donahue, K. R. \& Wanat, M. A. Management of Anticoagulation with Impella ${ }^{\circledR}$ Percutaneous Ventricular Assist Devices and Review of New Literature. Journal of thrombosis and thrombolysis 48, 284-291, doi:10.1007/s11239-019-01837-6 (2019).

10. Kappetein, A. P. et al. Updated standardized endpoint definitions for transcatheter aortic valve implantation: the Valve Academic Research Consortium-2 consensus document. J Am Coll Cardiol 60, 1438-1454, doi:10.1016/j.jacc.2012.09.001 (2012).

11. Mehran, R. et al. Standardized bleeding definitions for cardiovascular clinical trials: a consensus report from the Bleeding Academic Research Consortium. Circulation 123, 2736-2747, doi:10.1161/circulationaha.110.009449 (2011).

12. Wood, D. A. et al. Pivotal Clinical Study to Evaluate the Safety and Effectiveness of the MANTA Percutaneous Vascular Closure Device. Circ Cardiovasc Interv 12, e007258, doi:10.1161/circinterventions.119.007258 (2019).

13. Reynolds, H. R. \& Hochman, J. S. Cardiogenic shock: current concepts and improving outcomes. Circulation 117, 686-697, doi:10.1161/CIRCULATIONAHA.106.613596 (2008).

14. Freund, A. et al. Frequency and Impact of Bleeding on Outcome in Patients With Cardiogenic Shock. JACC Cardiovasc Interv 13, 1182-1193, doi:10.1016/j.jcin.2020.02.042 (2020).

15. Szem, J. W. et al. High-risk intrahospital transport of critically ill patients: safety and outcome of the necessary "road trip". Crit Care Med 23, 1660-1666, doi:10.1097/00003246-199510000-00009 (1995).

16. Moccetti, F. et al. Reduction of MANTA-associated vascular complications after implementation of key insights on failure mechanisms. Catheter Cardiovasc Interv, doi:10.1002/ccd.29696 (2021).

17. Ferreiro, J. L. et al. Use of Impella Recover LP 2.5 in elective high risk percutaneous coronary intervention. Int J Cardiol 145, 235-237, doi:10.1016/j.ijcard.2009.08.003 (2010).

18. Abaunza, M. et al. Incidence and prognosis of vascular complications after percutaneous placement of left ventricular assist device. J Vasc Surg 62, 417-423, doi:10.1016/j.jvs.2015.03.040 (2015).

19. O'Neill, W. W. et al. The current use of Impella 2.5 in acute myocardial infarction complicated by cardiogenic shock: results from the USpella Registry. J Interv Cardiol 27, 1-11, doi:10.1111/joic.12080 (2014).

20. Lauten, A. et al. Percutaneous left-ventricular support with the Impella-2.5-assist device in acute cardiogenic shock: results of the Impella-EUROSHOCK-registry. Circ Heart Fail 6, 23-30, doi:10.1161/CIRCHEARTFAILURE.112.967224 (2013).

21. Chehab, O. et al. Incidence and clinical outcomes of nosocomial infections in patients presenting with STEMI complicated by cardiogenic shock in the United States. Heart Lung 49, 716-723, 
doi:10.1016/j.hrtlng.2020.08.008 (2020).

22. Karatolios, K., Hunziker, P. \& Schibilsky, D. Managing vascular access and closure for percutaneous mechanical circulatory support. European Heart Journal Supplements 23, A10-A14, doi:10.1093/eurheartj/suab002 (2021).

\section{Figures}
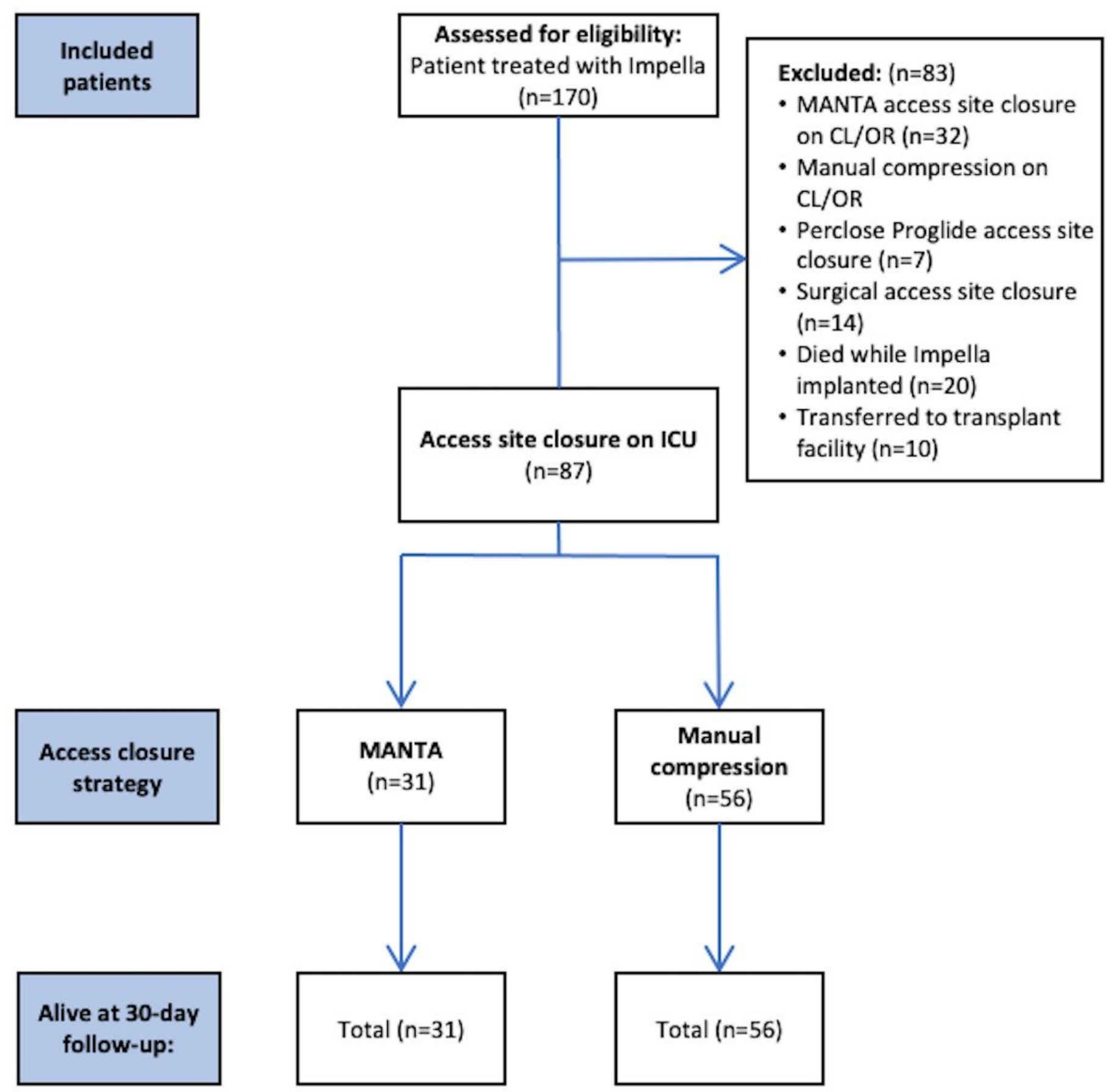

Figure 1 
Study flow chart

MCS = Mechanical circulatory support; ICU = Intensive care unit; $C L=$ Catheterization laboratory; $O R=$ Operating room.

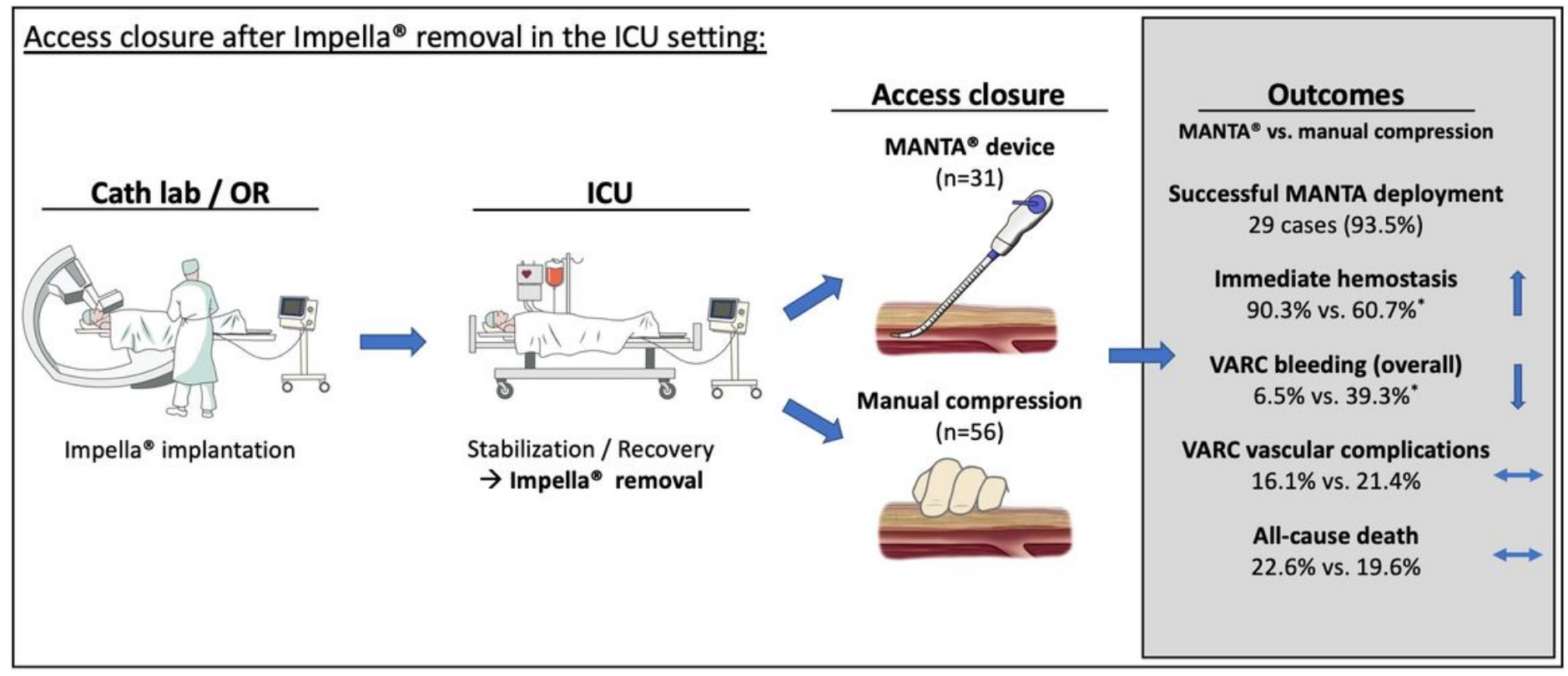

Figure 2

Access closure after Impella ${ }^{\circledR}$ removal in the ICU setting

$I C U=$ Intensive care unit; $O R=$ Operating room .

* Indicates a statistically significant difference $(p<0.05)$

\section{Supplementary Files}

This is a list of supplementary files associated with this preprint. Click to download.

- SupplementalTable1Narrativesofmajorcomplications.docx 